\title{
Energy Efficiency Optimization in Wireless Sensor Network Using Modified DRINA
}

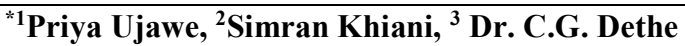 \\ ${ }^{*} 1,2$ Department of IT, GHRCEM, Pune \\ ${ }^{3}$ Director, Academic Staff, Nagpur \\ *Email: priya.ujawe@gmail.com
}

\author{
Received: $17^{\text {th }}$ January 2019, Accepted: $13^{\text {th }}$ February 2019, Published: $30^{\text {th }}$ June $_{2019}$
}

\begin{abstract}
Energy efficiency is an important parameter in wireless sensor network. To optimize the energy efficiency in wireless sensor network different methods are available. We have been proposed a new approach to reduce the energy in clustering nodes using data aggregation technique. The proposed Cluster-based modified data aggregation routing in-network algorithm (M-DRINA) is implemented for energy saving in wireless sensor network. M-DRINA periodically changes the cluster head and it select node with highest energy as a cluster head. It also helps to extend the lifetime of sensor networks and avoid the failures of wireless sensor network.
\end{abstract}

\section{Keywords}

In-Network Aggregation, Energy Efficiency, Wireless Sensor Networks (WSN), M-DRINA.

\section{Introduction}

A Wireless Sensor Network (WSN) is an electronic device that sense natural or physical conditions, for example, weight, movement, temperature, sound, vibration, or poisons at distinctive areas. WSN have many applications, for example, homeland security, environmental monitoring, communications, and manufacturing. Sensor node is device that is energy consumed and large connected with the measure of accumulated information, since correspondence is frequently the most costly action as far as energy. Many algorithm and protocols are design to reduce the energy in WSN. In addition, WSNs are information driven systems that for the most part deliver a lot of data that should be directed, regularly in a multihop fashion, toward a sink hub, which functions as gateway to an observing center (Fig. 1). Towards this situation, routing play important role in the information gathering process[6] .

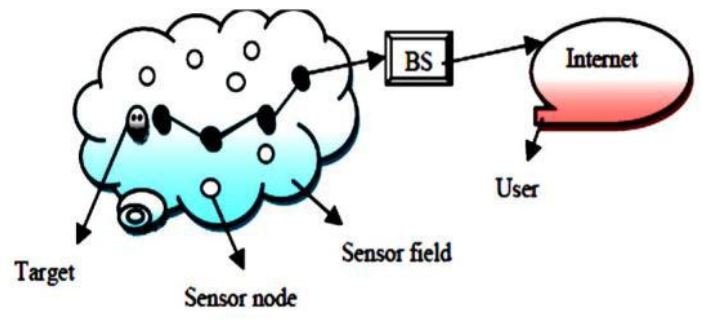

\section{Literature Survey}

Figure 1: Block Diagram for Wireless Sensor Network

In literature survey many protocols and algorithm was proposed to optimize the energy in wireless sensor network. Data driven is one of the techniques available in wireless sensor network for energy efficiency. This technique again divided into two parts A. In-Network aggregation B. Data aggregation

\section{A. In-Network Aggregation}

In the scenario of WSNs, the extraordinary approaches refers to in-community records aggregation through which intermediate nodes forward statistics packets toward the sink node even as combining the records combined from unique supply nodes. The design of a data aggregation aware routing protocol is the main component for in -network data aggregation. The possibility of the In-Network Aggregation is to aggregate the data to source close node rather than transmitting every detected esteem through the whole system [1]. 


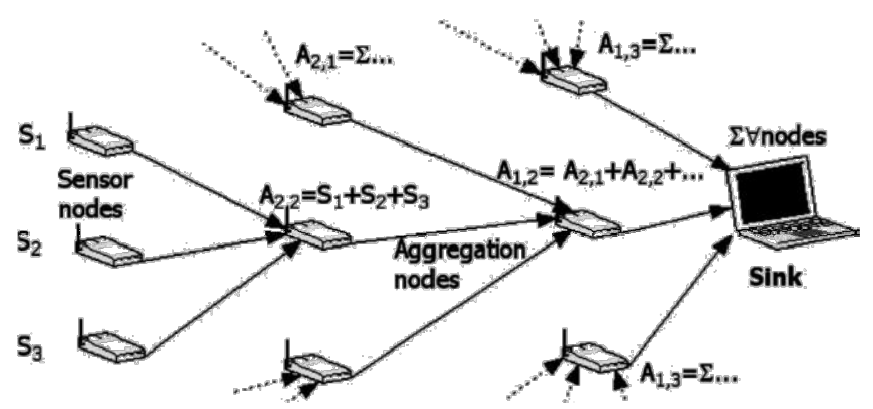

Figure 2: In-Network Aggregation Principle

\section{B. Data Aggregation}

Data aggregation technique again having subpart like tree based, cluster based, hybrid data aggregation

1) Tree Based Data Aggregation Approach: Protocols in this approach are usually based on a tree hierarchy of the nodes in the network where an intermediate node performs data aggregation process and defines a perfect direction for data flow from source node to sink node.

2) Cluster Based Data Aggregation Approach: Hierarchical organization of nodes is located on this method. Cluster is the collections of nodes. Inside the cluster unique node decided on as a cluster head which is chargeable for information aggregation and forwarded to the sink node. LEACH (Low-strength Adaptive Clustering Hierarchy) is the cluster-based totally network and statistics-aggregation protocol [9].

DRINA (data Routing for In-community Aggregation) is cluster-based set of rules . The DRINA algorithm works in 3 phases. In segment 1, sensor nodes build the hop tree to the sink node and the hop tree that get utilized by coordinators for facts forwarding purposes. In phase 2 cluster formations and cluster-head election is carried out the various nodes that discover the prevalence of a new event inside the network sooner or later, phase three is chargeable for both putting in a new course for the reliable turning in of packets and updating the hop tree [6].

\section{Proposed System}

The proposed system is optimizing the energy efficiency in clustering nodes using modified Data Routing for In-Network Aggregation (M-DRINA) . For multi hop communication cluster head required more energy. Using proposed system the energy for multi hop communication can be reduce. In Existing DRINA depending on hop counts cluster head are selected while in proposed M-DRINA depending upon remaining energy of nodes cluster heads are selected.

\section{A. Mathematical Model}

Energy consumption model is for multihop communication

$\varepsilon(n)=c(n) \times h(n) \times e(n)$

where $c(n)$ is the range of transmitted bit. And $h(n)$ is the common wide variety of hops for the transmission. e (n) is strength intake to transmit single bit. each node sends records only to cluster node (i.e. if $\mathrm{h}(\mathrm{n})=1)$ so electricity consumption through character node ni in time $\mathrm{t}$ is given by means of,

$\varepsilon\left(n_{i}\right)=c\left(n_{i}\right) \times 1 \times e\left(n_{i}\right) \times s r_{i} \times t$

Where sri Sampling rate of node ni in samples per second, $t$ is time in seconds

$\varepsilon(\mathrm{ch})=h(\mathrm{ch}) \times \sum_{i=0}^{n}(\varepsilon(\mathrm{ni}))$

Where $\mathrm{h}(\mathrm{ch})$ is number of hops for transmission from cluster head to sink node and $\mathrm{n}$ is number of nodes in cluster.

$\varepsilon_{\mathrm{t}}(\mathrm{ch})=t \times h(c h) \times \sum_{i=0}^{n}\left(\varepsilon\left(n_{i}\right)\right)$

In this proposed approach bunch head is chosen occasionally in view of outstanding vitality of hub, each hub persuade opportunity to be bunch set out toward some period, i.e in period t every node is cluster head for given time $(\mathrm{t} / \mathrm{n})$ or $(\mathrm{t}-\mathrm{t} / \mathrm{n})$ time as normal node .energy consumption by each node in time $\mathrm{t}$ is

$\varepsilon\left(n_{i}\right)=c\left(n_{i}\right) \times 1 \times e\left(n_{i}\right) \times s r_{i} \times\left(t-\frac{t}{n}\right)+\frac{t}{n} \times h(c h) \times \sum_{=0}^{n}\left(\varepsilon\left(n_{i}\right)\right)$ 
Proposed Cluster Formation Algorithm:

if node is sink node

cluster node $=$ self address

cluster Energy = self remaining energy

hops to $\sin \mathrm{k}=0$

else

cluster node $=$ broadcasts address

cluster Energy $=-1$

ii. if node is sink node

iii. else

a) $\quad$ RREQ( Seqno, nodeID, remaining Energy, hops to sink)
a) Wait for RREQ Packet.

iv. If RREQ packet received from neighbor nodes

a) if cluster node $=$ self then

i) cluster node $=$ Node Id in RREQ packet

ii) cluster energy = remaining energy in RREQ packet

b) else

i) if RREQ packet has hops to $\operatorname{sink}<$ hops to sink

(1) cluster node $=$ Node Id in RREQ packet

(2) cluster energy = remaining energy in RREQ packet

ii) else

(1) if RREQ packet has hops to sink = hops to sink

(2) If RREQ packet has remaining energy > cluster Energy then

(a) $\quad$ node $=$ Node Id in RREQ packet

(b) cluster energy = remaining energy in RREQ packet

else

(a) neglect RREQ packet

v. Repeat step 6 for every RREQ. Send Acknowledgement to cluster

\section{Experiment Result}

For experimental result we used OMNET++ simulator. Table 1 contain parameter list of implementation

\begin{tabular}{|c|c|c|}
\hline Sr. No. & Parameter & Value \\
\hline 1 & Network Area & $200 \mathrm{~m}$ \\
\hline 2 & Network Life Time & $500 \mathrm{~s}$ \\
\hline 3 & Nodes & 20 \\
\hline 4 & Packet Rate & 10 \\
\hline
\end{tabular}

Table 1: Parameter Table 


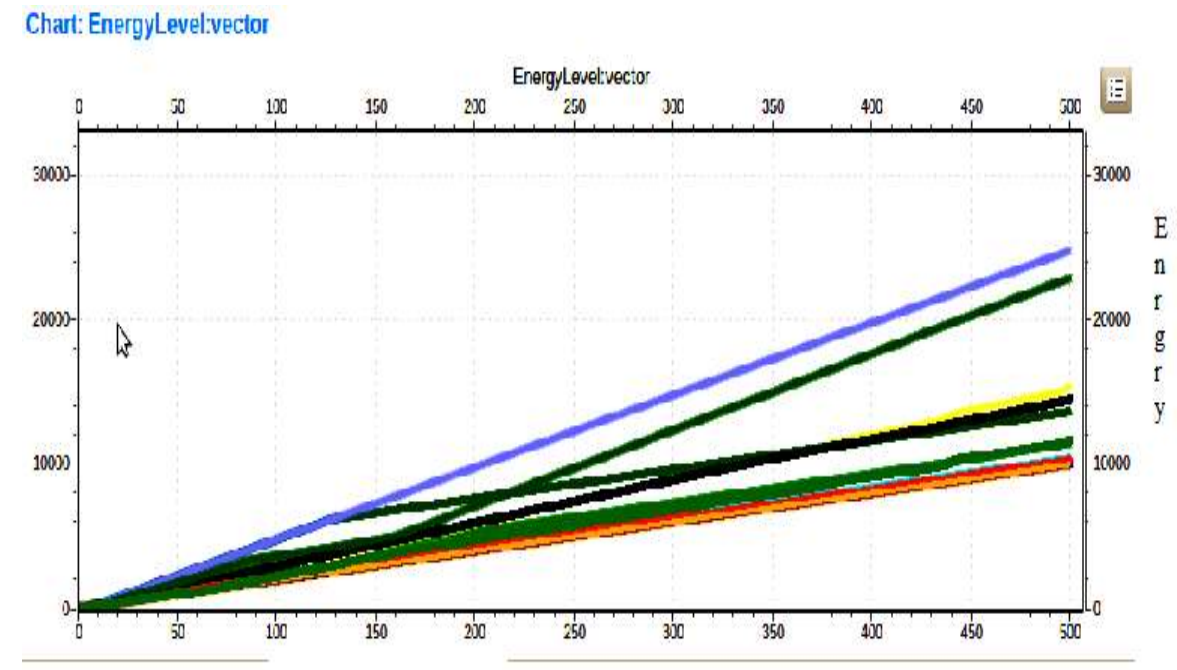

Time in Second

Figure 3: Energy Level in Existing DRINA

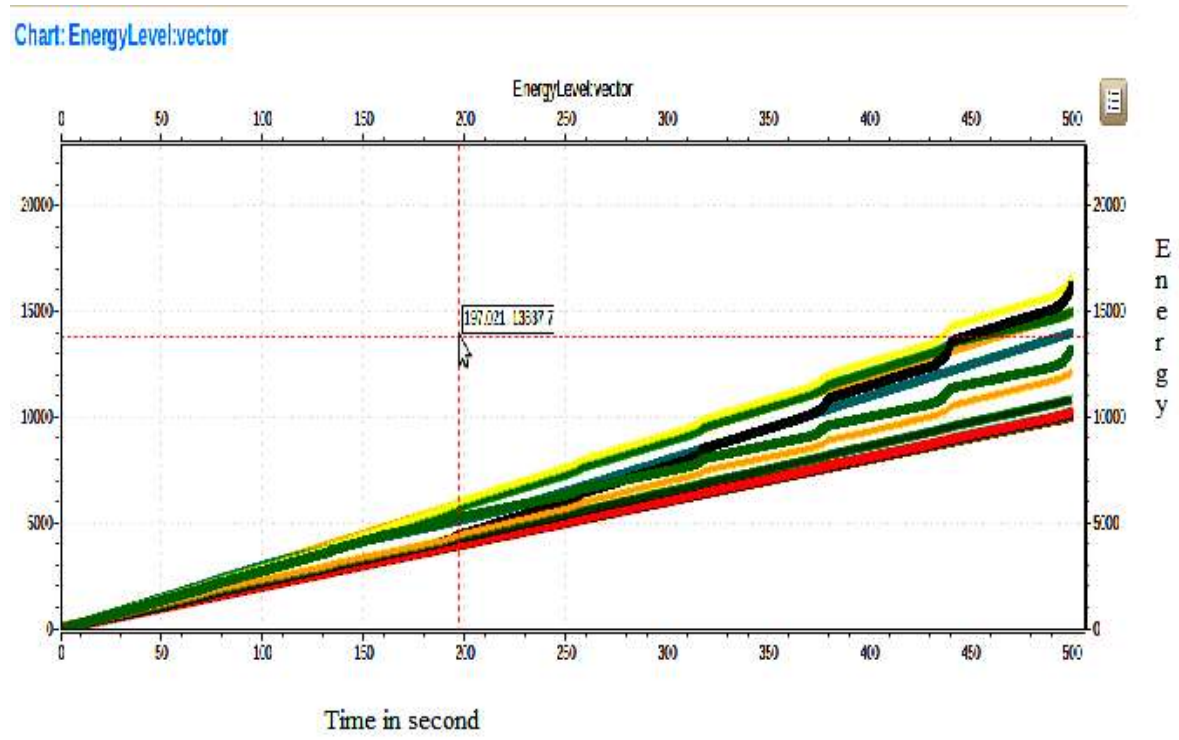

Figure 4: Energy Level in Proposed M-DRINA

For proposed system Network Life Time is 500s as cluster head is periodically changed and node with highest remaining power become cluster head. Energy of all nodes used almost equally.

Table 2 shows comparison between MDRINA \& DRINA for energy consumed using different parameters.

\begin{tabular}{|l|c|c|c|c|c|c|c|c|}
\hline Parameter & $\begin{array}{c}\text { M- } \\
\text { DRINA }\end{array}$ & $\begin{array}{c}\text { Existing } \\
\text { DRINA }\end{array}$ & $\begin{array}{c}\text { M- } \\
\text { DRINA }\end{array}$ & $\begin{array}{c}\text { Existing } \\
\text { DRINA }\end{array}$ & $\begin{array}{c}\text { M- } \\
\text { DRINA }\end{array}$ & $\begin{array}{c}\text { Existing } \\
\text { DRINA }\end{array}$ & $\begin{array}{c}\text { M- } \\
\text { DRINA }\end{array}$ & $\begin{array}{c}\text { Existing } \\
\text { DRINA }\end{array}$ \\
\hline Network Area & 200 & 200 & 500 & 500 & 500 & 500 & 500 & 500 \\
\hline No. of Nodes & 10 & 10 & 15 & 15 & 20 & 20 & 20 & 20 \\
\hline Packet Rate & 10 & 10 & 10 & 10 & 15 & 15 & 10 & 10 \\
\hline Cluster Head & 5 & 4 & 4 & 3 & 6 & 2 & 10 & 6 \\
\hline
\end{tabular}

Table 2: Comparison between MDRINA \& DRINA 


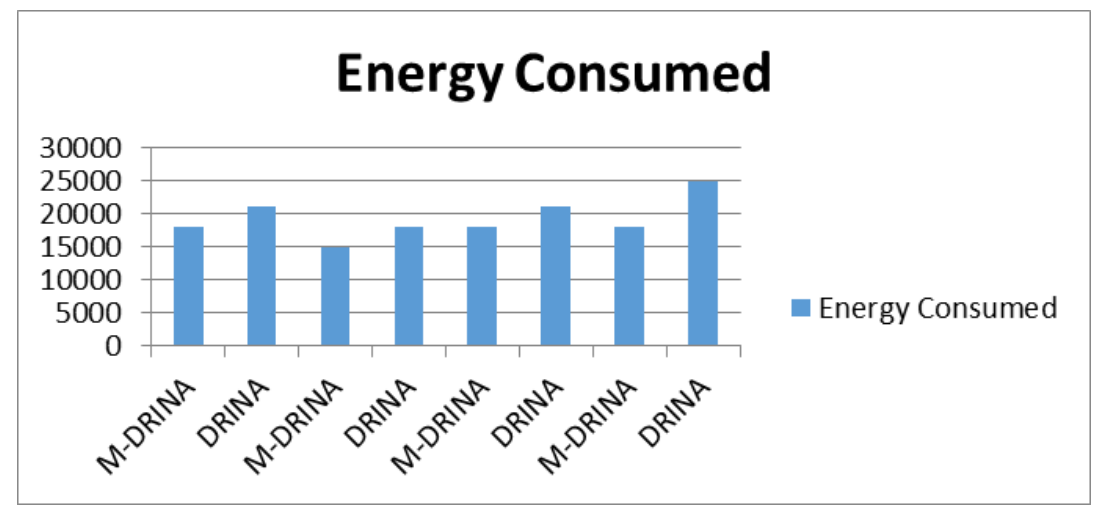

Fig. 5. Energy Consumption Comparison between MDRINA and DRINA

\section{Conclusion}

The proposed Cluster-based modified data aggregation routing in-network algorithm (DRINA) is implemented for energy saving in wireless sensor network. As compared to existing DRINA proposed MDRINA optimize $20-30 \%$ energy.

\section{Acknowledgment}

We are thankful to the Department of Information technology and G. H. Raisoni College of Engineering and Management, Wagholi, Pune, for providing necessary facilities to carry out this work. I would like to thank Ms. Simran Khiani of the Department of Information Technology at GHRCEM, Pune for valuable suggestions and guidance.

\section{References}

[1] G.M. Joshi, B.M. Patil, "Data Routing In-Network Aggregation for Wireless Sensor Network", IJCA March 2016

[2] Aditi C.Jadhav, Rashmi V. Telang, Akriti R.Verma, Prof. Kavita S. Kumavat, "Wireless Sensor Network using DRINA”, IJRITCC 2015

[3]Pei Zhiqiang, Xu Changqing , "A Max-Energy-Utilization Deployment Strategy in Wireless Sensor Networks", IEEE International Conference on Computational Science and Engineering, 2011 IEEE

[4]Rakesh Bisht, Dr Naveen Chauhan "A Traffic-Load Adaptive Power-Conserving MAC Protocol for Wireless Sensor Networks”, ICCCNT'12, 26th 28th July 2012, Coimbatore, India, IEEE-20180

[5]Kiran Maraiya, Kamal Kant, Nitin Gupta "Architectural Based Data Aggregation Techniques in Wireless Sensor Network: A Comparative Study”, International Journal on Computer Science and Engineering (IJCSE) Vol. 3 No. 3 Mar 2011

[6]Leandro Villas, Azzedine Boukerche1, Heitor S. Ramos "DRINA: A Lightweight and Reliable Routing Approach for in-Network Aggregation in

Wireless Sensor Networks", IEEE 2013

[7]Kemal Akkaya and Mohamed Younis, Moustafa Youssef "Efficient Aggregation of Delay-Constrained Data in Wireless Sensor Networks", IEEE, 2005

[8]Ruihua Zhang, Lei Ju, Zhiping Jia, Xin Li ,’Energy Efficient Routing Algorithm for WSNs Via Unequal Clustering", 2012 IEEE 14th International Conference on High Performance Computing and Communications

[9]Parul Bakaraniya, Shital Mehta, "Features of WSN and Various Routing Techniques For WSN: A survey", IJRET, 2012

[10]Steffen Peter , Krzysztof Piotrowski and Peter Langend orfer, “In-Network-Aggregation as Case Study for a Support Tool Reducing the Complexity of Designing Secure Wireless Sensor Networks”, IEEE 2008 